\title{
La intención emprendedora y su relación con las características personales y del contexto de las mujeres universitarias
}

The entrepreneurial intention and its correlation with personal characteristics and the external context of university women

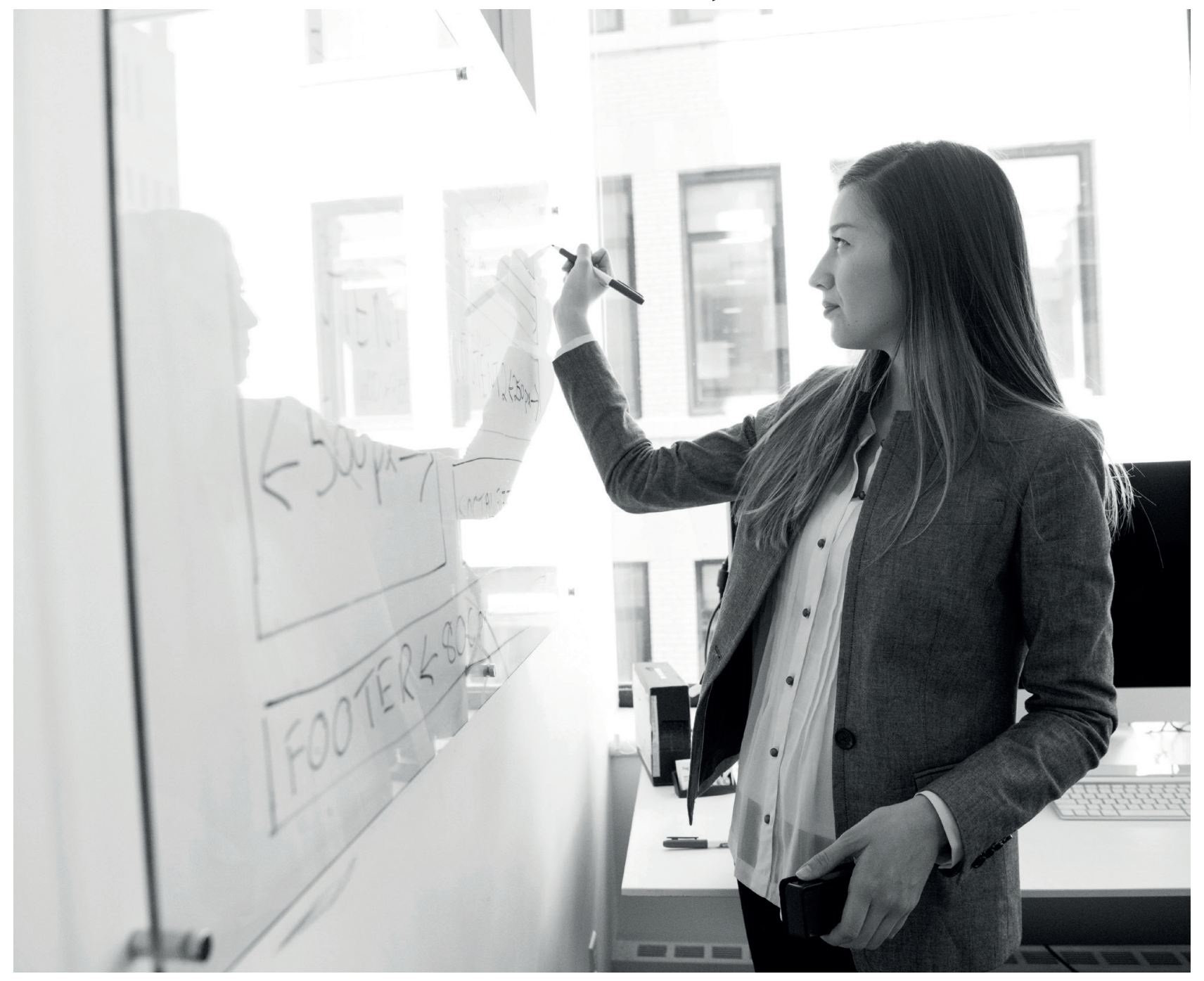




\title{
La intención emprendedora y su relación con las características personales y del contexto de las mujeres universitarias ${ }^{1}$
}

\author{
The entrepreneurial intention and its correlation with personal \\ characteristics and the external context of university women \\ Youseline Garavito-Hernández², Sebastián García-Méndez³, Johana Andrea Calderón- \\ Campos ${ }^{4}$
}

Artículo recibido en septiembre 25 de 2020; artículo aceptado en noviembre 13 de 2020

\begin{abstract}
Este artículo puede compartirse bajo la licencia Licencia Creative Commons Atribución-NoComercial-Compartir Igual 4.0 Internacional y se referencia usando el siguiente formato: Garavito-Hernández, Y., García-Méndez, S. y Calderón-Campos, J. (2021). La intención

emprendedora y su relación con las características personales y del contexto de las mujeres universitarias. I+D Revista de Investigaciones, 16(1), 238-253. http://dx.doi.org/10.33304/revinv.v16n1-2021019.
\end{abstract}

\begin{abstract}
Resumen
El objetivo de este trabajo es analizar la intención emprendedora en el marco de las características personales y factores del entorno de las mujeres del curso académico denominado Proyecto emprendedor, de los programas presenciales de cuatro facultades de una universidad en Bucaramanga, Colombia. La metodología se estructuró bajo un enfoque cuantitativo y tuvo un diseño no experimental, de corte transversal y explicativo. En tal sentido, se aplicó como fuente de información una encuesta conformada por 33 preguntas relacionadas con los factores objeto de estudio a 147 estudiantes, lo que permitió realizar un análisis descriptivo y correlacional. Los resultados revelan que existen diferencias significativas en la relación entre la intención emprendedora y las características personales y del entorno de las estudiantes de las cuatro facultades.
\end{abstract}

Palabras clave: emprendimiento, estudiantes, intención emprendedora, universidades.

\section{Abstract}

The objective of this work in progress is to analyze the entrepreneurial intention within the framework of the personal characteristics and factors of the environment of women of a University in Bucaramanga, Colombia. The methodology is structured under a quantitative approach and has a non-experimental, cross-sectional and explanatory design. In this sense, a survey consisting of 33 questions related to the factors under study was applied to 147 students. The

\footnotetext{
${ }_{1}$ Artículo de investigación, de tipo correlacional, de corte transversal, con enfoque cuantitativo, resultado de un proyecto de investigación terminado perteneciente al área de ciencias sociales, subárea gestión empresarial. Financiado por la Universidad de Investigación y Desarrollo (UDI) (Bucaramanga, Colombia). Dirección: calle 9 n. $23-55$, PBX: 6352525. Fecha de inicio: febrero de 2020. Fecha de finalización: noviembre de 2020.

2 Ph. D. en Dirección de Empresas, docente investigador del grupo de investigación PORTER de la Universidad de Investigación y Desarrollo (Bucaramanga, Colombia). Dirección: calle 9 n. 23-55, PBX: 6352525. ORCID ID: https://orcid.org/0000-0002-1644-9959. Correo electrónico institucional: ygaravito2@udi.edu.co.

${ }^{3}$ Magíster en Administración. Docente investigador del grupo de investigación SINERGIA de la Universidad de Investigación y Desarrollo (Bucaramanga, Colombia). Dirección: calle 9 n. 23-55, PBX: 6352525. ORCID ID: https://orcid.org/0000-0002-6077-0248. Correo electrónico institucional: sgarcia15@udi.edu.co.

${ }^{4}$ Magíster en Administración. Docente investigador del grupo de investigación PORTER de la Universidad de Investigación y Desarrollo (Bucaramanga, Colombia). Dirección: calle 9 n. 23-55, PBX: 6352525. ORCID ID: https://orcid.org/0000-0002-4008-4099. Correo electrónico institucional: jcalderon20@udi.edu.co.
} 
Youseline Garavito-Hernández, Sebastián García-Méndez, Johana Andrea Calderón-Campos

La intención emprendedora y su relación con las características personales y del contexto de las mujeres universitarias

results reveal that there are significant differences in the relationship between the entrepreneurial intention and the personal characteristics and the environment of the students of the four faculties.

Keywords: entrepreneurial intention, entrepreneurship, university students, universities

\section{Introducción}

El emprendimiento es un proceso de creación de nuevas empresas y se considera como el promotor del desarrollo económico de un país, ya que impulsa la innovación, la competitividad organizacional e incrementa la productividad (Ovalles-Toledo et al., 2018). En relación con su estudio, en los últimos años muchas investigaciones han empleado diseños metodológicos basados en revisión bibliográfica, análisis de casos, y pocos utilizan estadísticas para correlacionar variables (Forero-Bernal y Durán-Duarte, 2019; Santiago Martínez y Márquez Rodríguez, 2018). Este fenómeno igualmente se presenta en los trabajos de emprendimiento y género, en los cuales se evidencian muchas investigaciones comparativas con poca profundidad (Henry et al., 2016).

Bajo estas premisas, es de relevancia estudiar los factores vinculados a la intención emprendedora de las mujeres, ya que hoy en día todavía se evidencia una discriminación hacia ellas cuando laboran en ambientes liderados por hombres. En este contexto, el emprendimiento se está convirtiendo en una opción para desarrollarse como profesionales, obtener ingresos y enfrentar condiciones de discriminación (Mun y Jung, 2018).

Además, es necesario resaltar que, a pesar de los avances significativos de la mujer como emprendedora, aún existe un techo de cristal donde ellas pueden mirar hacia arriba, pero les es difícil cruzar. Por ello, es de relevancia identificar y seguir profundizando en aquellos factores relacionados con su intención emprendedora, con el fin de generar recomendaciones que promuevan el emprendimiento (Chirinos Araque et al., 2018). Entre los factores que requieren un mayor estudio es la educación, ya que provee de herramientas necesarias a las mujeres para hacer realidad sus proyectos de negocio (Ortiz Casallas, 2017)

Porconsiguiente, la educación en las universidades basada en el desarrollo de competencias en emprendimiento contribuye al fortalecimiento e incremento de la intención emprendedora en los estudiantes, especialmente en las mujeres, al promover comportamientos empresariales que les permiten superar el techo de cristal (Diaz Bretones y Radrigán, 2018). En síntesis, fortalecer las habilidades y competencias de las universitarias en temas de emprendimiento es crucial para que se empoderen en el ámbito laboral y económico al materializar sus ideas de negocios, lo que logra la supervivencia empresarial de sus iniciativas a lo largo del tiempo.
Es así que hoy en día se evidencian trabajos que estudian las variables inherentes en la intención emprendedora de los estudiantes, como los factores asociados a las características personales y del entorno del emprendedor; por ejemplo, la necesidad de logro, el locus de control, la creatividad, la autoestima, la capacidad de asumir riesgos, la experiencia previa, la formación, el background familiar, entre otros (García-Cabrera et al., 2015)

Este artículo de investigación se centra en estudiar las variables que se relacionan con la intención emprendedora de las mujeres que cursan un programa de pregrado de una universidad en Bucaramanga, Colombia. Esto con el fin de poder identificar aquellas características personales de las estudiantes y factores del entorno que necesitan ser afianzados en el sistema de educación superior en pro del fortalecimiento de la intención emprendedora de las mujeres universitarias. Para ello, en primer lugar, se enuncian los estudios previos y se proponen las hipótesis de investigación; posteriormente, se explica la metodología de investigación y se dan a conocer los resultados y conclusiones.

\section{Fundamentación teórica}

El emprendedor se caracteriza por ser un visionario que conduce un proceso de cambio, descubre y crea nuevas oportunidades (Cuervo García et al., 2005). También se define como una persona innovadora que crea incertidumbre y perturba el equilibrio del mercado (Schumpeter, 1934). Algunos estudios se han enfocado en analizar los factores que influyen en su intención de emprender, y se concluye que la preferencia que tiene una persona en ser emprendedora depende de ciertas variables (Galindo y Herrera, 2017; Zambrano-Vargas y Vázquez-García, 2019).

Entre los factores que influyen en la intención emprendedora están las características personales, que difieren de acuerdo con el individuo. Por dar algunos ejemplos, está la asunción al riesgo, experiencia, trabajo en equipo, capacidad de escucha, locus de control, formación, resolución de problemas, necesidad de logro, pensamiento a largo plazo, disciplina, resiliencia, inteligencia emocional, comunicación, delegación, entre otros. También están los factores del entorno, como la familia, redes de apoyo, universidades, oportunidades productivas, factores socioculturales, institucionales y políticos (Guzmán y Santos, 2001; Laukkanen, 2000; Pabón, 2011; Van Gelderen et al., 2006). 
Youseline Garavito-Hernández, Sebastián García-Méndez, Johana Andrea Calderón-Campos La intención emprendedora y su relación con las características personales y del contexto de las mujeres universitarias

Según informe GEM (2017), la tasa de actividad emprendedora en Colombia alcanza el 18,9\%. De este porcentaje, el $60 \%$ son emprendedores nacientes, y el 40 \%, nuevos empresarios (Gómez Núñez et al., 2017, p. 5). Igualmente, se evidencia que el emprendimiento de oportunidad (14,5\%) tiene mayor grado en comparación con el emprendimiento de necesidad (3,5 \%) (Gómez Núñez et al., 2017). Así mismo, este informe presenta una disminución en la diferencia porcentual de los emprendimientos de género durante el periodo 20132017 , y se evidencia un 19,2 \% para los hombres y un $18,2 \%$ para las mujeres. Esto demuestra un aumento de la iniciativa emprendedora del género femenino en la última década.

En este contexto, se destacan los estudios enfocados en la intención emprendedora en las mujeres (Berdugo Cotera y Gámex Gutiérrez, 2015), ya que ellas representan aproximadamente la mitad de la población mundial, pero solo el $16 \%$ crean negocios y tienen éxito (García et al., 2018; Mun y Jung, 2018). Esto se debe a diversas causas. Algunos autores concluyen que existe una dificultad en la adquisición de recursos, lo que genera como resultado un menor rendimiento en sus ideas emprendedoras (OECD, 2017; Robb, 2002). Otros argumentan que se debe a que muchas de ellas tienen una aversión al riesgo mayor que los hombres y tienden a adoptar un crecimiento lento y constante en sus negocios (Marlow y Patton, 2005; Tinkler et al., 2015).

En este orden de ideas, investigaciones afirman que estas diferencias pueden ser debido a una medición no apropiada del rendimiento; por ejemplo, las mujeres se caracterizan por crear empresas más pequeñas que los hombres. Del mismo modo, reconocen que la motivación y el balance entre trabajo y vida personal influyen en el tamaño que pueda tener una empresa y su velocidad de crecimiento (Cliff, 1998; García et al., 2018; Kepler y Shane, 2007; Sabarwal y Terrell, 2008).

Estos hallazgos, entre otros, concuerdan con la teoría liberal feminista, al argumentar que si las mujeres tuvieran igualdad de oportunidades que los hombres en educación, experiencia laboral, entre otros, podrían tener comportamientos similares en relación con el crecimiento y tamaño de sus iniciativas de negocios (Naldi et al., 2019). Trabajos basados en esta perspectiva demuestran que los hombres poseen un alto nivel de autoafirmación, de autoexpansión y necesidad de dominar; y las mujeres tienen altos niveles de desinterés, preocupación por los demás y sensibilidad interpersonal, características que influyen en la financiación, el tamaño y tipo de emprendimientos y los rendimientos futuros (Clancy, 2007; Eagly y Wood, 1991).

Por otra parte, autores que han estudiado el emprendimiento femenino enfocan sus resultados basados en las teorías de género y estado de bienestar, al demostrar que en muchos hogares los hombres tienen ingresos económicos mayores que sus parejas, a pesar de tener la misma experiencia y formación. Se suma el hecho de que en muchos hogares son ellas las encargadas de realizar las labores domésticas. Estos elementos promueven la intención emprendedora de muchas mujeres al poder compaginar las labores domésticas, el cuidado de sus hijos y mejorar sus ingresos (Mun y Jung, 2018; Thébaud, 2010).

Este fenómeno ha originado en los últimos años un aumento del emprendimiento femenino, a pesar de que la tasa sigue siendo baja en comparación con los hombres en algunas regiones del mundo. Según Bosma y Kelley (2019), en el ámbito mundial en países como Arabia Saudita, Qatar y Madagascar la tasa de emprendimiento de las mujeres supera la de los hombres. Algunas de las razones para emprender están relacionadas con su intención de hacer una diferencia en el mundo y ganarse la vida, porque los trabajos son escasos. En el caso de los hombres, emprenden para construir una riqueza, mejorar los ingresos o continuar con la tradición familiar (Bosma et al., 2020). Así, existen diferentes motivaciones para emprender (Pinillos Costa et al., 2007), y el Global Entrepreneurship Monitor (GEM) distingue dos: "[...] los emprendimientos generados por razones asociadas a la necesidad y aquellos generados por la identificación de una oportunidad" (Gómez Núñez et al., 2017, p. 25).

En este orden de ideas, la actual crisis económica producto de la enfermedad de la COVID-19 da origen al aumento de la intención emprendedora de las personas a nivel mundial, y por ende los futuros emprendimientos se convierten en "un instrumento para dinamizar las economías y promover la flexibilidad del tejido productivo a través de la creatividad y la innovación, generando cambios estructurales en la economía local, regional y nacional" (Martínez y Rubio Bañón, 2020).

En Latinoamérica, la tasa de emprendimiento femenino es alta si se compara con Europa y Asia. Según datos de Bosma et al. (2020), la actividad empresarial de los países latinoamericanos supera el $10 \%$ en 21 de las 50 economías de la región. En el caso de las mujeres colombianas, se caracterizan por su continua búsqueda de independencia. Según el Departamento Administrativo Nacional de Estadísticas (2018) la mayor posición ocupacional para las mujeres $(41,1 \%)$ fue trabajadora por cuenta propia.

En este panorama, la educación se convierte en una variable de relevancia para las mujeres, al permitirles desarrollar competencias en emprendimiento necesarias para favorecer la iniciativa emprendedora y llegar a tener éxito en sus ideas de negocios (Ortiz Casallas, 2017). Es por ello que la formación en las universidades es fundamental porque contribuye al fortalecimiento e incremento de la intención emprendedora al promover comportamientos empresariales para superar el techo 
Youseline Garavito-Hernández, Sebastián García-Méndez, Johana Andrea Calderón-Campos

La intención emprendedora y su relación con las características personales y del contexto de las mujeres universitarias

de cristal que afrontan muchas de ellas (Diaz Bretones y Radrigán, 2018).

Algunos estudios enfocados en analizar la intención emprendedora en universitarios han encontrado que las mujeres dan más importancia a las recompensas personales (crecimiento personal, reconocimiento público o probar que se es capaz de hacerlo) y menos a las económicas (Rueda et al., 2013). Igualmente, han demostrado que la educación es de relevancia para ellas por aportar a su proyecto de vida. Así, la universidad se percibe como un espacio para formar, entrenar y capacitar a las estudiantes, porque les permiten potenciar sus competencias (genéricas y técnicas) para aprovechar las oportunidades del mercado y favorecer la creación de nuevos negocios (Ortiz Casallas, 2017).

En este orden de ideas, tal como se mencionó en la introducción, este trabajo de investigación tiene como objetivo indagar cómo se relacionan ciertas características personales de las mujeres universitarias y de su entorno con la intención emprendedora, con el ánimo de proponer recomendaciones en emprendimiento femenino para las instituciones de educación superior (IES).

\section{Hipótesis de trabajo}

Hipótesis 1: Las características personales de las mujeres universitarias se relacionan con su intención emprendedora.

Hipótesis 2: Los factores del entorno de las mujeres universitarias se relacionan con su intención emprendedora.

\section{Metodología}

\section{Tipo de estudio}

El estudio se estructuró bajo un enfoque cuantitativo y un diseño no experimental de corte transversal. Además, es de tipo correlacional, ya que su interés se centra en analizar la relación entre dos o más variables, pero en ningún momento explica que una sea la causa de la otra (Hernandez Sampieri et al., 2006).

\section{Población y muestra}

La población está conformada por 147 estudiantes (mujeres) del curso académico Proyecto emprendedor, pertenecientes a 13 programas presenciales de cuatro facultades. Se eligió este curso académico porque los estudiantes desarrollan como proyecto final un plan de negocios con potencial implementación en un futuro cercano. En esta línea, la Tabla 1 presenta los porcentajes para cada grupo poblacional.
Tabla 1

Curso académico proyecto emprendedor de los programas presenciales participantes

\begin{tabular}{|c|c|c|c|}
\hline Facultades & $\%$ & Programa académico & $\%$ \\
\hline \multirow{2}{*}{$\begin{array}{l}\text { Ciencias } \\
\text { Administrativas, } \\
\text { Económicas y } \\
\text { Contables }\end{array}$} & \multirow{2}{*}{27} & Administración Empresas & 23 \\
\hline & & Negocios Internacionales & 4 \\
\hline \multirow{4}{*}{$\begin{array}{l}\text { Comunicación, } \\
\text { Artes y Diseño }\end{array}$} & \multirow{4}{*}{31} & Comunicación Social & 16 \\
\hline & & Publicidad Marketing Digital & 2 \\
\hline & & Diseño Industrial & 3 \\
\hline & & Diseño Gráfico & 10 \\
\hline \multirow{4}{*}{ Ingenierías } & \multirow{4}{*}{30} & Ingeniería Civil & 0 \\
\hline & & Ingeniería Industrial & 23 \\
\hline & & Ingeniería Electrónica & 3 \\
\hline & & Ingeniería de Sistemas & 4 \\
\hline \multirow{3}{*}{$\begin{array}{l}\text { Ciencias Sociales y } \\
\text { Humanas }\end{array}$} & \multirow{3}{*}{59} & Criminalística & 16 \\
\hline & & Derecho & 7 \\
\hline & & Psicología & 36 \\
\hline
\end{tabular}

Fuente: Autores.

\section{Participantes}

La población objeto de estudio está conformada por mujeres universitarias con edades que oscilan entre los $18-25$ años $(83,7 \%)$, estado civil soltera $(95,9 \%$ ) y sin hijos $(87,8 \%)$. Además, el $83 \%$ de las estudiantes proviene de un estrato social medio, y el $40 \%$ de ellas está cursado un programa académico de la Facultad de Ciencias Sociales y Humanas: Psicología (24,5 \%); Criminalística $(10,9 \%)$ y Derecho (4,8 \%); el 20,40 \%, de la Facultad de Ingenierías (Ingeniería Industrial, un 15,6 \%; Ingeniería de Sistemas, 2,7\%, e Ingeniería Electrónica, 2,0\%). Se evidencia que la mayoría de las estudiantes que cursan proyecto emprendedor en el primer semestre del 2020 son del programa académico de Psicología.

\section{Instrumento de medición}

El instrumento que se utilizó para la recolección de información fue una encuesta de 33 preguntas tendientes a evaluar la intención emprendedora, las características personales y factores del entorno de las estudiantes universitarias. Para construir el instrumento se utilizaron estudios previos de intención emprendedora y la información del Global Entrepreneurship Monitor (Garavito-Hernández et al., 2020). Se validó a través del método Delphi, el cual se basa en el trabajo con jueces expertos en el tema que se está investigando, con el fin de corroborar que se incluyen todos los elementos representativos de lo que se quiere medir (George Reyes y Trujillo Liñan, 2018). 
Youseline Garavito-Hernández, Sebastián García-Méndez, Johana Andrea Calderón-Campos La intención emprendedora y su relación con las características personales y del contexto de las mujeres universitarias

\section{Confiabilidad del instrumento}

Se realizó un análisis de fiabilidad, para conocer la confiabilidad del instrumento de medición. En tal sentido, el método más utilizado es el Alfa de Cronbach, el cual oscila entre 0 y 1 . El valor mínimo aceptable para el coeficiente es $(0,7)$. Particularmente, para este estudio fue de $(0,8)$, por tanto, el instrumento es confiable.

\section{Medición de las variables}

\section{Variable dependiente Intención emprendedora:}

Dentro del estudio del emprendimiento en jóvenes universitarios, autores como Valencia Arias et al. (2016) consideran la intención emprendedora como:

El estado de la mente en el que la atención de una persona se centra en el cumplimiento de un objetivo, en este caso la creación de una empresa o negocio lo cual tiene cierta influencia en las acciones a tomar por el individuo para llegar a dicho objetivo. (p. 883)

Esta variable se considera dicotómica y toma el valor de 1 si la estudiante tiene una percepción positiva hacia la intención emprendedora, y de 0 , en caso contrario.

\section{Variables independientes}

Características personales del emprendedor: Son cualidades esenciales que conforman los atributos básicos del talento humano, tales como la capacidad para resolver problemas, cognición, necesidad de logro, eficiencia, locus de control interno, conocimiento, formación, habilidades, experiencia, coste de oportunidad, edad y estado civil, entre otros (Sastre, 2013). Variables dicotómicas que toman el valor de 1 si la estudiante se identifica con el factor, y de 0 , en caso contrario.

Los factores del entorno del emprendedor: El entorno del emprendedor es considerado como una de las condiciones del contexto en el que se desenvuelve el emprendedor; cuenta con características particulares como la familia, redes de apoyo y situación social y económica actual (Kurniawan et al., 2019). Variables dicotómicas que toman el valor de 1 si la estudiante se identifica con el factor, y de 0 , en caso contrario.

Tabla 2

Variables dependientes e independientes

\begin{tabular}{cc}
\hline & Variable dependiente \\
\hline Variable & Intención emprendedora \\
Dimensiones & Intención emprendedora \\
\hline & Variable independiente \\
\hline
\end{tabular}

\begin{tabular}{cc}
\hline Variable & $\begin{array}{c}\text { Características personales } \\
\text { Asumir riesgos, experiencia, trabajo en equipo, } \\
\text { capacidad de escucha, formación, locus de } \\
\text { control interno, resolución de problemas, pen- } \\
\text { samiento a largo plazo, coste de oportunidad, } \\
\text { necesidad de logro, inteligencia emocional, } \\
\text { disciplina, resiliencia, respeto, comunicación, } \\
\text { delegación, pasión. }\end{array}$ \\
Variable & $\begin{array}{r}\text { Variables demográficas } \\
\text { Dimensiones }\end{array}$ \\
Variables & $\begin{array}{c}\text { Sexo, edad, estado civil, estrato social, hijos. } \\
\text { Factores del entorno personal del empren- } \\
\text { dedor }\end{array}$ \\
Dimensiones & Familia y amigos, redes de apoyo, situación \\
económica y social actual (COVID-19).
\end{tabular}

Fuente: Autores.

\section{Correlación entre variables}

Según Roy-García et al. (2019), la correlación entre las variables permite conocer la fuerza de asociación entre dos variables cuantitativas u ordinales, la cual se expresa por medio de un determinado coeficiente, cuyo intervalo es entre -1 y 1 . En este ámbito, es importante mencionar que las dos metodologías más usadas son el coeficiente de correlación de Pearson y el Rho de Spearman, cuya utilización depende de la estructura de los datos. Para esta investigación se utilizó el Rho de Spearman.

\section{Resultados}

\section{Análisis descriptivo}

Los resultados de la presente investigación se enmarcan en los estadísticos descriptivos para las diferentes preguntas, teniendo presentes las variables y las dimensiones. En esta línea, se hace necesario mencionar la codificación planteada para los grupos poblacionales objeto de estudio:

- Facultad de Ciencias Económicas, Administrativas y Contables: F1

- Facultad de Comunicación, Artes y Diseño: F2

- Facultad de Ingenierías: F3

- Facultad de Ciencias Sociales y Humanas: F4

El análisis de frecuencias es presentado en las gráficas $1,2,3,4$ y 5 . En tal sentido, se recalca la presencia de la variable dependiente, a saber, intención emprendedora; y las independientes, características personales y factores del entorno. Las dimensiones por cada variable dependiente e independiente se relacionan en el Anexo 1.

La variable dependiente tiene inherentemente vinculada 1 dimensión, la cual está asociada a las siguientes preguntas:

- Soy una persona que prefiere crear un negocio que buscar un empleo: $\mathrm{P} 1$ 
Youseline Garavito-Hernández, Sebastián García-Méndez, Johana Andrea Calderón-Campos

La intención emprendedora y su relación con las características personales y del contexto de las mujeres universitarias

- Me surgen ideas para emprender un negocio: P2

- Al terminar la carrera profesional tengo planeado crear un negocio: P3

- Quiero emprender un negocio mientras estudio en la universidad: P4

En este contexto, los resultados de la variable dependiente se presentan en la Figura 1, y se permite observar que las mujeres adscritas a la Facultad de Comunicación, Artes y Diseño tienen una predisposición positiva a la creación de negocios, y es este un elemento característico del perfil del educando de las disciplinas inherentes (Sánchez García et al., 2017). Por su parte, se reconoce que las estudiantes de la Facultad de Ciencias
Económicas, Administrativas y Contables tienen en mayor medida competencias orientadas al reconocimiento de las necesidades o insatisfacciones de un determinado nicho de mercado, lo cual les posibilita generar con mayor facilidad ideas de negocio (Borjas de Xena, 2012).

De igual manera, se visualiza que las alumnas vinculadas a la Facultad de Ciencias Económicas, Administrativas y Contables tienen contemplado crear un negocio durante o al finalizar la formación de pregrado. Esto es vinculable a los perfiles de ingreso y egreso en un alto porcentaje de las instituciones de educación superior del país, caso contrario al de los demás programas académicos (Sánchez García et al., 2017).

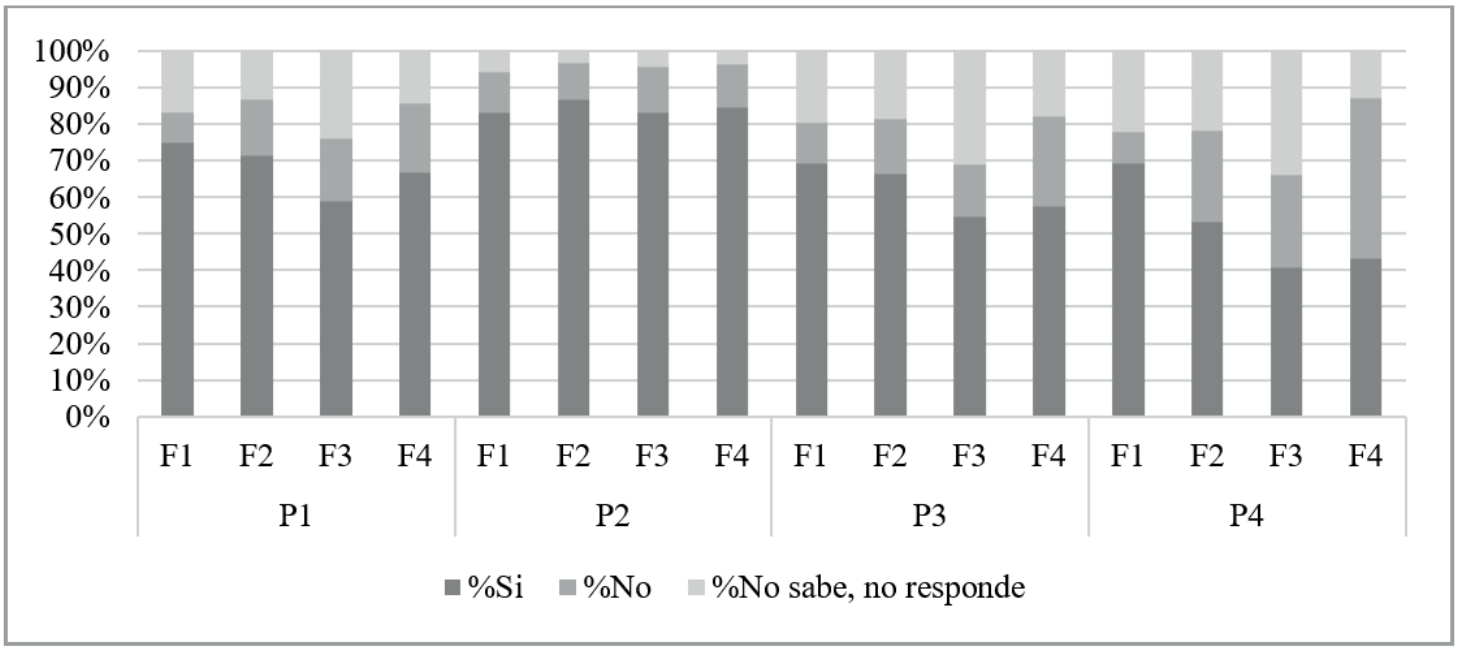

Figura 1. Intención Emprendedora en estudiantes universitarios. Fuente: Autores.

Por otro lado, la variable independiente "Características personales" se analizó por medio de las dimensiones referentes a asumir riesgos, experiencia, trabajo en equipo, capacidad de escucha, formación (capacitación y conocimiento), locus de control interno, resolución de problemas, pensamiento a largo plazo, coste de oportunidad, necesidad de logro, inteligencia emocional, disciplina, resiliencia, respeto, comunicación, delegación y pasión.

De acuerdo con los resultados, la Figura 2 muestra que cerca del 97,22 \% de las estudiantes de la Facultad de Ciencias Económicas, Administrativas y Contables están dispuestas asumir un riesgo para crear un nuevo negocio. Un porcentaje menor se aprecia para el resto de las entrevistadas, que oscilan entre $85 \%$ y el $90 \%$. En relación con la experiencia en emprender un negocio, la mayoría de las estudiantes manifiestan que tienen poca experiencia $(66,67 \%$ para la Facultad de Ciencias
Económicas, Administrativas y Contables; $70 \%$ para la Facultad de Comunicación, Artes y Diseño; 80,28 \% para la Facultad de Ingenierías, y 68,24\% para la Facultad de Ciencias Sociales y Humanas). A pesar de ello, las encuestadas de la Facultad de Ingenierías $(66,20 \%)$ y de la Facultad de Ciencias Sociales y Humanas (72,94\%) consideran que tienen la capacitación en temas de emprendimiento. Asimismo, más del $88 \%$ de las personas encuestadas consideran que tienen las habilidades para trabajar en equipo y capacidad de escucha; por lo tanto, son más receptivas con las opiniones de otras personas con relación a sus ideas de negocio. Por último, más de la mitad de las alumnas manifiestan que tienen los conocimientos técnicos necesarios para elaborar un determinado producto o servicio. 
Youseline Garavito-Hernández, Sebastián García-Méndez, Johana Andrea Calderón-Campos La intención emprendedora y su relación con las características personales y del contexto de las mujeres universitarias

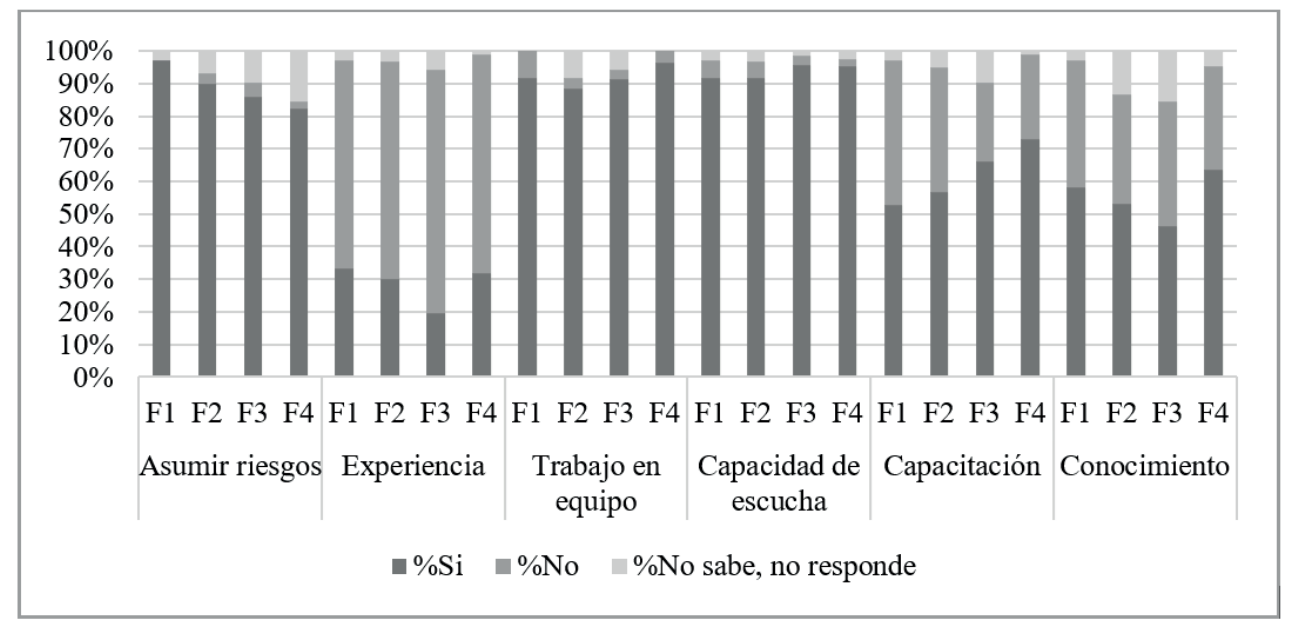

Figura 2. Características personales. Fuente: Autores.

Por otro lado, se observa en las Figuras 3 y 4 que más del $80 \%$ de las estudiantes se consideran capaces de sacar adelante un negocio, suelen buscar alternativas de solución cuando tienen un problema para emprender y, en su mayoría, se describen como personas capaces de controlar sus emociones ante las dificultades. Además, las encuestadas creen que montar un negocio es un proyecto para toda la vida y valoran que es para obtener beneficios económicos. En este orden de ideas, las mujeres de este estudio tienen la percepción de que ser emprendedoras mejoraría su calidad de vida.

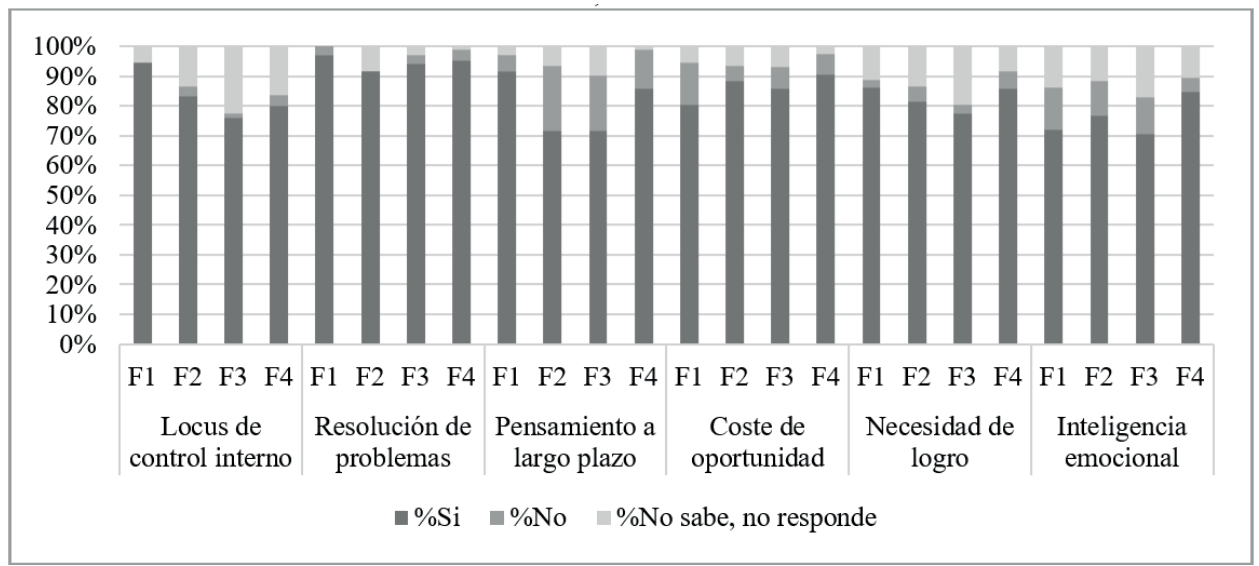

Figura 3. Características personales. Fuente: Autores.

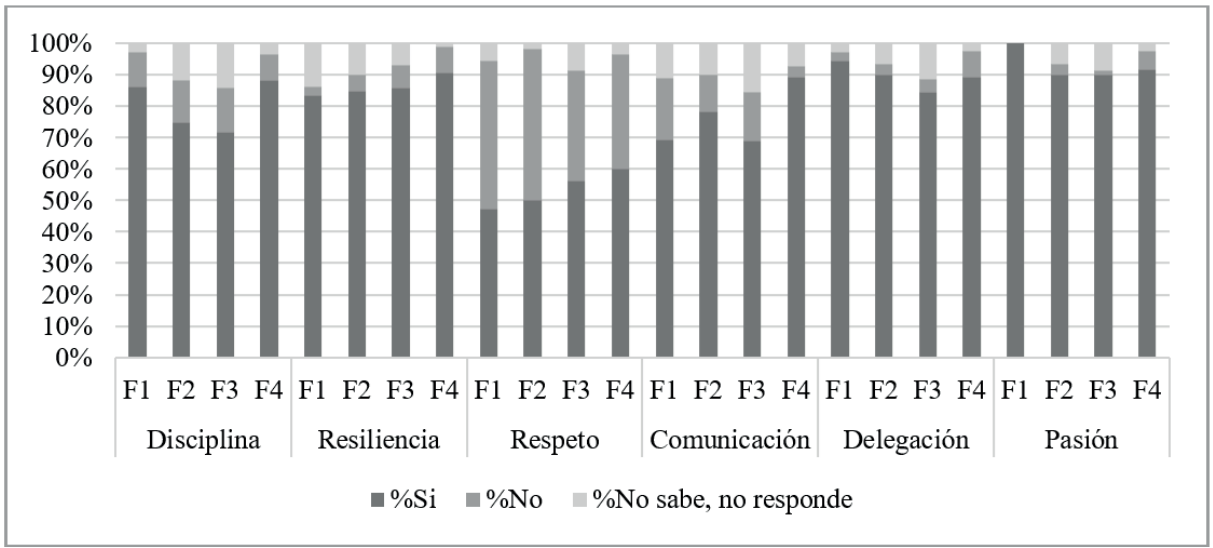

Figura 4. Características personales. Fuente: Autores. 
Youseline Garavito-Hernández, Sebastián García-Méndez, Johana Andrea Calderón-Campos

La intención emprendedora y su relación con las características personales y del contexto de las mujeres universitarias

Con respecto a la Figura 5, relacionada con los factores del entorno, como la familia (familiares emprendedores y apoyo familiar), las redes de apoyo y la situación actual, el análisis descriptivo permite evidenciar que el apoyo de la familia es importante para la intención emprendedora de las alumnas, y la situación actual no es un obstáculo para emprender. En cuanto a las redes de apoyo, según lo manifestado por las universitarias, podría decirse que es un área de oportunidad como escenario de motivación en este ecosistema de emprendimiento.

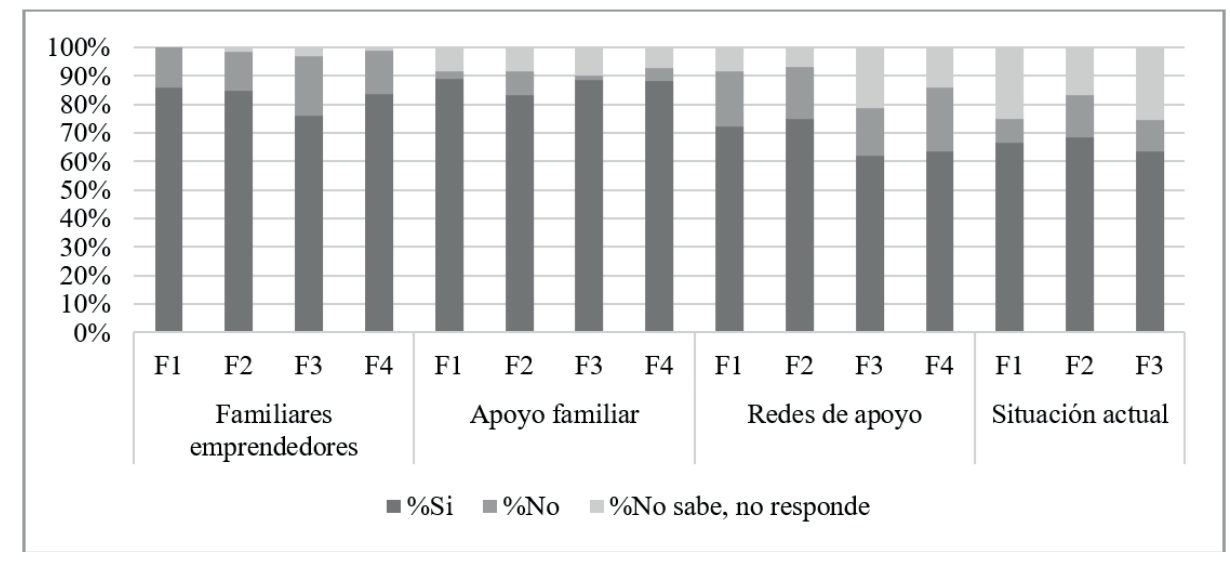

Figura 5. Factores del entorno. Fuente: Autores.

\section{Análisis correlacional}

El análisis de correlación se realizó bajo el coeficiente de Spearman, teniendo en cuenta los valores de significancia de la prueba Kolmogorov-Smimov o Shapiro-Wilk, en razón al tamaño de la muestra de las estudiantes adscritas a las facultades objeto de estudio. Se resalta que en la dimensión inherente a la resolución de problemas no se puede emitir ningún juicio particular de relación en la Facultad de Ciencias Económicas, Administrativas y Contables y en la Facultad de Ingenierías, teniendo en cuenta que la totalidad de las estudiantes se identifican con la afirmación. En otros términos estadísticos, la desviación estándar y varianza es igual a cero, caso similar en la dimensión de pasión para la Facultad de Ciencias Económicas, Administrativas y Contables. La Tabla 3 presenta la codificación de las dimensiones. El análisis contempla los resultados de las dimensiones con un Factor de inflación de la varianza (FIV) menor a 10 para las variables independientes.

Tabla 3

Codificación de las dimensiones

\begin{tabular}{cc}
\hline Nombre largo & Nombre corto \\
\hline Intención emprendedora & D1 \\
Asumir riesgos & D2 \\
Experiencia & D3 \\
Trabajo en equipo & D4 \\
Capacidad de escucha & D5 \\
Formación & D6 \\
Locus de control interno & D7 \\
Resolución de problemas & D8 \\
Pensamiento a largo plazo & D9 \\
\hline
\end{tabular}

\begin{tabular}{cc}
\hline Coste de oportunidad & D10 \\
Necesidad de logro & D11 \\
Inteligencia emocional & D12 \\
Disciplina & D13 \\
Resiliencia & D14 \\
Respeto & D15 \\
Comunicación & D16 \\
Delegación & D17 \\
Pasión & D18 \\
Familia & D19 \\
Redes de apoyo & D20 \\
Situación actual & D21
\end{tabular}

Fuente: Autores.

El análisis de correlación de las estudiantes de la Facultad de Ciencias Económicas, Administrativas y Contables se presenta en la Tabla 4. Los resultados permiten visualizar que la intención emprendedora se correlaciona positivamente con la dimensión disciplina; es decir, si la estudiante es una persona ordenada y cumple ciertas normas o reglas en función de un resultado, será mayor su intención de crear un negocio. Por otro lado, si estas mujeres tienen familiares emprendedores, mayores serán sus conocimientos para elaborar un producto con el cual podrían crear una nueva idea y se sentirán más capacitadas para atreverse a emprender. De esta misma manera, se consideran personas que tienen la facilidad de delegar actividades $y$, por ende, mayor capacidad de transmitir con claridad sus ideas y de controlar sus emociones. Asimismo, estas mujeres no se rinden ante las dificultades e intentan de nuevo. Esto se relaciona con su facilidad de asumir riesgos y ser capaces de sacar un negocio adelante. 
Youseline Garavito-Hernández, Sebastián García-Méndez, Johana Andrea Calderón-Campos La intención emprendedora y su relación con las características personales y del contexto de las mujeres universitarias

Tabla 4

Correlación de Spearman: Facultad Ciencias Económicas, Administrativas y Contables

\begin{tabular}{|c|c|c|c|c|c|c|c|c|c|c|c|c|c|c|c|c|c|c|c|c|}
\hline & FIV & D1 & D2 & D3 & D4 & D5 & D6 & D7 & D9 & D10 & D11 & D12 & D13 & D14 & D15 & D16 & D17 & D19 & D20 & D21 \\
\hline D1 & & 1,0 & & & & & & & & & & & & & & & & & & \\
\hline D2 & 8,9 & $-0,1$ & 1,0 & & & & & & & & & & & & & & & & & \\
\hline D3 & 2,1 & 0,3 & 0,2 & 1,0 & & & & & & & & & & & & & & & & \\
\hline D4 & 4,1 & $-0,2$ & $-0,1$ & $-0,4$ & 1,0 & & & & & & & & & & & & & & & \\
\hline D5 & 10,9 & $-0,2$ & 0,6 & 0,0 & 0,4 & 1,0 & & & & & & & & & & & & & & \\
\hline D6 & 4,6 & 0,3 & 0,2 & 0,2 & $-0,1$ & $-0,2$ & 1,0 & & & & & & & & & & & & & \\
\hline D7 & 3,8 & 0,3 & 0,0 & 0,2 & $-0,1$ & $-0,1$ & 0,2 & 1,0 & & & & & & & & & & & & \\
\hline D9 & 2,5 & 0,3 & 0,0 & $-0,3$ & $-0,1$ & $-0,1$ & 0,2 & 0,0 & 1,0 & & & & & & & & & & & \\
\hline D10 & 3,2 & $-0,1$ & 0,4 & $-0,1$ & 0,2 & 0,4 & $-0,1$ & $-0,1$ & $-0,1$ & 1,0 & & & & & & & & & & \\
\hline D11 & 5,6 & 0,3 & $-0,1$ & 0,3 & $-0,1$ & 0,3 & 0,0 & 0,6 & $-0,1$ & 0,1 & 1,0 & & & & & & & & & \\
\hline D12 & 3,4 & 0,4 & 0,3 & 0,0 & 0,1 & 0,3 & 0,2 & $-0,1$ & 0,3 & $-0,2$ & 0,0 & 1,0 & & & & & & & & \\
\hline D13 & 1,7 & 0,5 & $-0,1$ & 0,1 & $-0,1$ & $-0,1$ & 0,1 & $-0,1$ & $-0,1$ & 0,0 & 0,2 & 0,0 & 1,0 & & & & & & & \\
\hline D14 & 2,5 & 0,2 & 0,4 & 0,0 & $-0,1$ & 0,1 & 0,0 & 0,4 & $-0,1$ & 0,0 & 0,1 & 0,1 & 0,1 & 1,0 & & & & & & \\
\hline D15 & 4,8 & 0,1 & 0,2 & 0,0 & $-0,3$ & 0,1 & 0,3 & 0,2 & 0,2 & 0,2 & 0,1 & 0,2 & 0,0 & 0,1 & 1,0 & & & & & \\
\hline D16 & 5,1 & 0,1 & 0,4 & 0,0 & $-0,2$ & 0,1 & 0,0 & $-0,1$ & $-0,1$ & 0,4 & 0,1 & 0,4 & 0,3 & 0,2 & 0,4 & 1,0 & & & & \\
\hline D17 & 4,1 & 0,2 & $-0,1$ & 0,2 & $-0,1$ & $-0,1$ & 0,2 & $-0,1$ & $-0,1$ & $-0,2$ & 0,4 & 0,4 & 0,3 & $-0,1$ & 0,0 & 0,5 & 1,0 & & & \\
\hline D19 & 4,3 & 0,0 & 0,3 & 0,3 & $-0,2$ & 0,1 & 0,5 & 0,3 & $-0,1$ & $-0,1$ & 0,1 & 0,0 & $-0,2$ & 0,4 & $-0,1$ & $-0,1$ & $-0,2$ & 1,0 & & \\
\hline D20 & 2,9 & 0,4 & 0,3 & 0,2 & 0,1 & 0,0 & 0,3 & 0,3 & $-0,1$ & 0,2 & 0,3 & 0,1 & 0,2 & 0,3 & $-0,1$ & 0,2 & 0,1 & 0,4 & 1,0 & \\
\hline D21 & 8,1 & 0,4 & $-0,1$ & 0,0 & 0,1 & 0,3 & 0,2 & 0,3 & 0,3 & $-0,2$ & 0,5 & 0,3 & 0,0 & 0,1 & $-0,1$ & $-0,2$ & 0,1 & 0,4 & 0,3 & 1,0 \\
\hline
\end{tabular}

Nota: Las celdas sombreadas reflejan una correlación significativa en el nivel de 0,01 y 0,05 (bilateral). Fuente: Autores.

Por su parte, la Tabla 5 muestra el análisis de correlación de las estudiantes de la Facultad de Comunicación, Artes y Diseño. Los resultados permiten visualizar que la intención emprendedora es mayor en aquellas mujeres que tienen una mejor disposición a asumir riesgos y experiencia en crear negocios. Por otro lado, si ellas asumen ser capaces de sacar un negocio adelante, mayor será la aversión al riesgo; para ello, deben fortalecer su capacidad de solucionar problemas, ser disciplinadas, entusiastas y positivas.

Además, en este grupo de estudio se observa que cuanta mayor disciplina y resiliencia, mayor será la probabilidad de que busquen otras alternativas de solución si enfrentan algún problema con la puesta en marcha de su emprendimiento y de esta manera, logren empoderarse de su negocio para sacarlo adelante. Asimismo, los resultados demuestran que aquellas alumnas con familiares emprendedores tendrán una mayor experiencia en formar un negocio. Finalmente, tener amigos y socios emprendedores aumentará la probabilidad de que perciban su emprendimiento como un proyecto de vida; aumentará el gusto por trabajar en equipo, para que logren convertirse en personas disciplinadas en pro de sus metas.

Tabla 5.

Correlación de Spearman: Facultad Comunicación, Artes y Diseño

\begin{tabular}{|c|c|c|c|c|c|c|c|c|c|c|c|c|c|c|c|c|c|c|c|c|c|c|}
\hline & FIV & D1 & D2 & D3 & D4 & D5 & D6 & D7 & D8 & D9 & D10 & D11 & D12 & D13 & D14 & D15 & D16 & D17 & D18 & D19 & D20 & D21 \\
\hline D1 & & 1,0 & & & & & & & & & & & & & & & & & & & & \\
\hline D2 & 7,9 & 0,4 & 1,0 & & & & & & & & & & & & & & & & & & & \\
\hline D3 & 8,4 & 0,4 & 0,2 & 1,0 & & & & & & & & & & & & & & & & & & \\
\hline D4 & 2,0 & 0,0 & $-0,1$ & $-0,2$ & 1,0 & & & & & & & & & & & & & & & & & \\
\hline D5 & 3,5 & 0,1 & $-0,1$ & $-0,1$ & 0,4 & 1,0 & & & & & & & & & & & & & & & & \\
\hline D6 & 4,2 & 0,3 & 0,1 & 0,2 & 0,1 & 0,2 & 1,0 & & & & & & & & & & & & & & & \\
\hline D7 & 7,8 & 0,1 & 0,4 & 0,2 & $-0,1$ & $-0,1$ & 0,2 & 1,0 & & & & & & & & & & & & & & \\
\hline D8 & 6,0 & 0,2 & 0,6 & 0,1 & $-0,1$ & 0,0 & 0,2 & 0,7 & 1,0 & & & & & & & & & & & & & \\
\hline D9 & 4,1 & 0,2 & 0,2 & $-0,1$ & 0,2 & 0,2 & 0,2 & $-0,1$ & $-0,1$ & 1,0 & & & & & & & & & & & & \\
\hline D10 & 8,2 & $-0,3$ & $-0,1$ & 0,3 & 0,2 & 0,3 & 0,0 & $-0,1$ & $-0,1$ & $-0,2$ & 1,0 & & & & & & & & & & & \\
\hline D11 & 2,5 & 0,2 & $-0,1$ & 0,1 & 0,2 & 0,2 & 0,0 & $-0,1$ & $-0,1$ & 0,0 & 0,1 & 1,0 & & & & & & & & & & \\
\hline D12 & 10,1 & 0,1 & 0,6 & 0,0 & $-0,2$ & $-0,1$ & 0,3 & 0,5 & 0,3 & 0,2 & 0,0 & $-0,2$ & 1,0 & & & & & & & & & \\
\hline D13 & 4,3 & 0,1 & 0,4 & 0,3 & $-0,2$ & $-0,1$ & 0,0 & 0,5 & 0,4 & 0,0 & $-0,2$ & $-0,2$ & 0,3 & 1,0 & & & & & & & & \\
\hline D14 & 5,1 & 0,3 & 0,2 & 0,3 & $-0,1$ & $-0,1$ & 0,2 & 0,7 & 0,5 & $-0,2$ & $-0,1$ & 0,1 & 0,3 & 0,5 & 1,0 & & & & & & & \\
\hline D15 & 3,9 & 0,1 & 0,1 & $-0,2$ & 0,1 & 0,0 & 0,1 & 0,2 & 0,2 & 0,0 & $-0,4$ & $-0,3$ & 0,0 & 0,3 & 0,2 & 1,0 & & & & & & \\
\hline D16 & 4,5 & 0,0 & 0,2 & 0,1 & $-0,1$ & $-0,1$ & 0,0 & $-0,1$ & $-0,1$ & 0,3 & $-0,2$ & 0,0 & 0,2 & 0,0 & $-0,2$ & $-0,5$ & 1,0 & & & & & \\
\hline D17 & 2,5 & $-0,1$ & $-0,1$ & 0,1 & $-0,1$ & 0,0 & 0,2 & 0,0 & 0,0 & $-0,1$ & $-0,1$ & 0,4 & $-0,1$ & $-0,1$ & $-0,1$ & $-0,2$ & 0,4 & 1,0 & & & & \\
\hline D18 & 4,0 & 0,2 & 0,6 & 0,2 & $-0,1$ & $-0,1$ & 0,3 & 0,4 & 0,6 & 0,2 & $-0,1$ & $-0,1$ & 0,3 & 0,1 & 0,2 & 0,1 & $-0,1$ & $-0,1$ & 1,0 & & & \\
\hline D19 & 4,2 & 0,1 & 0,1 & 0,4 & $-0,2$ & 0,2 & 0,3 & 0,2 & $-0,1$ & $-0,2$ & 0,0 & 0,2 & 0,1 & 0,1 & 0,3 & $-0,3$ & 0,2 & 0,3 & 0,1 & 1,0 & & \\
\hline D20 & 2,4 & 0,3 & 0,1 & 0,0 & 0,4 & 0,2 & 0,1 & $-0,1$ & $-0,1$ & 0,5 & 0,1 & 0,0 & 0,1 & 0,0 & 0,1 & 0,1 & 0,0 & $-0,1$ & 0,1 & $-0,3$ & 1,0 & \\
\hline D21 & 5,7 & 0,2 & $-0,2$ & 0,2 & $-0,2$ & $-0,1$ & $-0,1$ & $-0,1$ & $-0,1$ & $-0,2$ & $-0,2$ & 0,2 & $-0,1$ & $-0,1$ & 0,3 & 0,0 & 0,0 & $-0,1$ & $-0,2$ & $-0,1$ & 0,1 & 1,0 \\
\hline
\end{tabular}

Nota: Las celdas sombreadas reflejan una correlación significativa en el nivel de 0,01 y 0,05 (bilateral). Fuente: Autores. 
Youseline Garavito-Hernández, Sebastián García-Méndez, Johana Andrea Calderón-Campos

La intención emprendedora y su relación con las características personales y del contexto de las mujeres universitarias

Por otra parte, en la Tabla 6 se puede evidenciar que no existe ninguna relación significativa entre las dimensiones (características personales y factores del entorno) y la intención emprendedora en la Facultad de Ingenierías. Este es un elemento potencialmente objeto de análisis en futuras investigaciones; particularmente se denota que las celdas sombreadas en color gris no se pueden contemplar, teniendo en cuenta el alto valor del factor de inflación de la varianza (FIV).

De igual manera, se percibe que existe una relación relevante entre el trabajo en equipo y la disposición a asumir riesgos en la población objeto de estudio, lo que se vincula con lo planteado por Gómez Mujica y Acosta Rodríguez (2003) en torno a la disposición natural de alcanzar los resultados planteados a priori, por medio de la cohesión y sinergia entre un grupo de personas, tengan estas pensamientos homogéneos o heterogéneos, pero basados en la complementariedad. Asimismo, se resalta una gran importancia de la comunicación, debido a la interacción con las dimensiones de trabajo en equipo, locus de control interno y disciplina. En tal sentido, Pimienta (2008) manifiesta el requerimiento latente de transmitir continuas ideas e información a los diversos stakeholders, con el propósito de lograr las metas temporales.

Tabla 6

Correlación de Spearman: Facultad Ingenierías

\begin{tabular}{|c|c|c|c|c|c|c|c|c|c|c|c|c|c|c|c|c|c|c|c|c|c|}
\hline & FIV & D1 & D2 & D3 & D4 & D5 & D6 & D7 & D9 & D10 & D11 & D12 & D13 & D14 & D15 & D16 & D17 & D18 & D19 & D20 & D21 \\
\hline D1 & & 1,0 & & & & & & & & & & & & & & & & & & & \\
\hline D2 & 15,5 & 0,4 & 1,0 & & & & & & & & & & & & & & & & & & \\
\hline D3 & 4,3 & 0,0 & 0,1 & 1,0 & & & & & & & & & & & & & & & & & \\
\hline D4 & 9,3 & 0,2 & 0,7 & 0,1 & 1,0 & & & & & & & & & & & & & & & & \\
\hline D5 & 4,3 & $-0,1$ & 0,0 & 0,1 & 0,0 & 1,0 & & & & & & & & & & & & & & & \\
\hline D6 & 2,5 & 0,0 & $-0,3$ & 0,1 & $-0,2$ & 0,2 & 1,0 & & & & & & & & & & & & & & \\
\hline D7 & 42,8 & 0,6 & 0,2 & 0,1 & $-0,1$ & $-0,1$ & 0,0 & 1,0 & & & & & & & & & & & & & \\
\hline D9 & 10,9 & 0,1 & 0,2 & $-0,1$ & 0,3 & 0,3 & 0,2 & 0,1 & 1,0 & & & & & & & & & & & & \\
\hline D10 & 32,5 & 0,3 & 0,3 & 0,2 & $-0,1$ & $-0,1$ & $-0,1$ & 0,5 & 0,2 & 1,0 & & & & & & & & & & & \\
\hline D11 & 11,0 & 0,2 & 0,4 & $-0,1$ & $-0,1$ & $-0,1$ & 0,1 & 0,1 & 0,1 & 0,5 & 1,0 & & & & & & & & & & \\
\hline D12 & 4,5 & 0,1 & 0,1 & 0,0 & $-0,1$ & 0,3 & $-0,2$ & 0,3 & 0,0 & 0,0 & 0,0 & 1,0 & & & & & & & & & \\
\hline D13 & 16,6 & 0,4 & $-0,1$ & $-0,1$ & $-0,1$ & $-0,1$ & 0,1 & 0,6 & 0,1 & $-0,1$ & $-0,1$ & 0,5 & 1,0 & & & & & & & & \\
\hline D14 & 4,5 & $-0,2$ & $-0,1$ & $-0,2$ & 0,0 & 0,0 & 0,0 & $-0,1$ & $-0,1$ & $-0,1$ & $-0,1$ & 0,1 & $-0,1$ & 1,0 & & & & & & & \\
\hline D15 & 4,3 & 0,3 & 0,1 & $-0,1$ & 0,2 & 0,2 & 0,1 & 0,2 & 0,6 & $-0,1$ & $-0,3$ & 0,1 & 0,4 & $-0,2$ & 1,0 & & & & & & \\
\hline D16 & 9,2 & 0,3 & 0,2 & 0,3 & 0,4 & $-0,1$ & 0,3 & 0,5 & 0,3 & 0,0 & $-0,2$ & 0,2 & 0,4 & $-0,1$ & 0,3 & 1,0 & & & & & \\
\hline D17 & 13,1 & 0,1 & $-0,1$ & 0,1 & 0,0 & 0,0 & 0,2 & 0,5 & 0,2 & $-0,1$ & $-0,1$ & 0,1 & 0,4 & $-0,1$ & 0,1 & 0,5 & 1,0 & & & & \\
\hline D18 & 7,3 & 0,2 & 0,0 & 0,1 & 0,0 & 0,0 & 0,2 & 0,3 & 0,3 & $-0,1$ & $-0,1$ & 0,3 & 0,6 & 0,0 & 0,2 & 0,4 & 0,7 & 1,0 & & & \\
\hline D19 & 8,1 & 0,2 & $-0,2$ & $-0,1$ & $-0,1$ & $-0,1$ & 0,1 & 0,2 & 0,0 & 0,4 & 0,3 & $-0,1$ & 0,1 & 0,4 & 0,0 & 0,1 & $-0,2$ & $-0,1$ & 1,0 & & \\
\hline D20 & 2,4 & $-0,1$ & $-0,2$ & $-0,1$ & $-0,2$ & $-0,2$ & $-0,1$ & 0,0 & $-0,3$ & $-0,3$ & 0,0 & 0,1 & 0,2 & 0,1 & $-0,1$ & $-0,1$ & 0,1 & $-0,2$ & 0,1 & 1,0 & \\
\hline D21 & 2,0 & 0,3 & 0,1 & 0,0 & $-0,1$ & 0,3 & 0,0 & $-0,1$ & 0,1 & $-0,1$ & 0,2 & 0,2 & 0,0 & 0,1 & 0,0 & $-0,2$ & 0,1 & 0,3 & $-0,1$ & 0,0 & 1,0 \\
\hline
\end{tabular}

Nota: Las celdas sombreadas reflejan una correlación significativa en el nivel de 0,01 y 0,05 (bilateral). Fuente: Autores.

Tabla 7

Correlación de Spearman: Facultad Ciencias Sociales y Humanas

\begin{tabular}{|c|c|c|c|c|c|c|c|c|c|c|c|c|c|c|c|c|c|c|c|c|c|c|}
\hline & FIV & D1 & D2 & D3 & D4 & D5 & D6 & D7 & D8 & D9 & D10 & D11 & D12 & D13 & D14 & D15 & D16 & D17 & D18 & D19 & D20 & D21 \\
\hline D1 & & 1,0 & & & & & & & & & & & & & & & & & & & & \\
\hline D2 & 1,8 & 0,2 & 1,0 & & & & & & & & & & & & & & & & & & & \\
\hline D3 & 1,4 & 0,1 & 0,1 & 1,0 & & & & & & & & & & & & & & & & & & \\
\hline D4 & 5,6 & 0,0 & 0,2 & 0,1 & 1,0 & & & & & & & & & & & & & & & & & \\
\hline D5 & 4,2 & $-0,1$ & 0,1 & 0,1 & 0,8 & 1,0 & & & & & & & & & & & & & & & & \\
\hline D6 & 1,6 & 0,0 & 0,2 & 0,2 & 0,0 & $-0,1$ & 1,0 & & & & & & & & & & & & & & & \\
\hline D7 & 1,4 & 0,2 & 0,2 & 0,2 & 0,1 & 0,1 & 0,2 & 1,0 & & & & & & & & & & & & & & \\
\hline D8 & 2,6 & 0,1 & 0,3 & 0,1 & 0,0 & $-0,1$ & 0,2 & 0,2 & 1,0 & & & & & & & & & & & & & \\
\hline D9 & 1,7 & 0,0 & 0,2 & 0,2 & 0,2 & 0,2 & 0,0 & 0,0 & 0,4 & 1,0 & & & & & & & & & & & & \\
\hline D10 & 1,7 & 0,2 & 0,2 & 0,0 & 0,3 & 0,2 & 0,0 & 0,1 & $-0,1$ & 0,3 & 1,0 & & & & & & & & & & & \\
\hline D11 & 1,6 & 0,2 & 0,0 & 0,2 & 0,2 & 0,1 & $-0,2$ & 0,2 & 0,1 & 0,1 & 0,0 & 1,0 & & & & & & & & & & \\
\hline D12 & 1,5 & $-0,1$ & 0,2 & 0,2 & $-0,1$ & $-0,1$ & 0,2 & 0,2 & 0,3 & 0,3 & 0,0 & 0,1 & 1,0 & & & & & & & & & \\
\hline D13 & 1,7 & 0,0 & 0,0 & 0,1 & 0,2 & 0,2 & $-0,1$ & 0,2 & 0,2 & 0,0 & $-0,1$ & 0,0 & 0,1 & 1,0 & & & & & & & & \\
\hline D14 & 1,6 & 0,2 & 0,0 & 0,0 & $-0,1$ & $-0,1$ & 0,0 & 0,2 & 0,2 & 0,0 & 0,1 & 0,0 & 0,0 & 0,2 & 1,0 & & & & & & & \\
\hline D15 & 1,6 & 0,0 & $-0,2$ & $-0,1$ & 0,0 & $-0,1$ & 0,0 & 0,1 & $-0,2$ & 0,0 & 0,0 & $-0,1$ & 0,1 & 0,2 & 0,2 & 1,0 & & & & & & \\
\hline D16 & 2,3 & $-0,1$ & $-0,1$ & 0,0 & $-0,1$ & 0,1 & 0,2 & 0,0 & $-0,1$ & 0,0 & 0,0 & $-0,2$ & 0,2 & $-0,1$ & 0,1 & $-0,1$ & 1,0 & & & & & \\
\hline D17 & 1,9 & $-0,2$ & $-0,1$ & 0,2 & 0,3 & 0,2 & 0,0 & 0,0 & $-0,1$ & $-0,1$ & $-0,1$ & $-0,1$ & 0,0 & 0,1 & $-0,1$ & 0,0 & 0,4 & 1,0 & & & & \\
\hline D18 & 2,3 & 0,1 & $-0,1$ & 0,2 & 0,3 & 0,2 & 0,0 & 0,1 & 0,2 & 0,3 & 0,3 & $-0,1$ & 0,2 & 0,1 & $-0,1$ & 0,2 & 0,0 & 0,1 & 1,0 & & & \\
\hline D19 & 1,7 & 0,1 & 0,3 & 0,2 & 0,1 & 0,0 & 0,3 & 0,2 & 0,4 & 0,1 & 0,0 & $-0,1$ & 0,3 & 0,3 & 0,0 & 0,0 & $-0,1$ & 0,0 & 0,1 & 1,0 & & \\
\hline D20 & 1,6 & 0,3 & $-0,1$ & 0,2 & 0,3 & 0,2 & 0,0 & 0,0 & 0,2 & 0,3 & 0,0 & 0,1 & 0,1 & 0,2 & 0,0 & $-0,1$ & 0,1 & 0,2 & 0,3 & 0,1 & 1,0 & \\
\hline D21 & 1,4 & 0,3 & 0,2 & 0,1 & 0,1 & 0,0 & $-0,1$ & 0,1 & 0,0 & 0,1 & $-0,1$ & 0,2 & 0,1 & 0,0 & 0,1 & $-0,1$ & $-0,1$ & 0,0 & $-0,2$ & 0,0 & 0,2 & 1,0 \\
\hline
\end{tabular}

Nota: Las celdas sombreadas reflejan una correlación significativa en el nivel de 0,01 y 0,05 (bilateral). Fuente: Autores. 
Youseline Garavito-Hernández, Sebastián García-Méndez, Johana Andrea Calderón-Campos La intención emprendedora y su relación con las características personales y del contexto de las mujeres universitarias

Para el caso de las estudiantes de la Facultad de Ciencias Sociales y Humanas, se puede identificar en la Tabla 7 que la intención emprendedora se relaciona con la situación actual provocada por la COVID-19, lo cual representa una oportunidad para emprender. Es decir, están dispuestas a emprender por necesidad, ya que,

[...] losemprendedoresmotivadosporlanecesidad son aquellos individuos que al momento de tomar la decisión de poner en marcha una empresa no disponían de otras oportunidades de trabajo, y esta nueva actividad empresarial es su única opción de generar ingresos. (Gómez Núñez et al., 2017)

Además, se identificó que aumentan su intención emprendedora cuando tienen un mayor número de amigos y contactos que puedan ser socios estratégicos si ellas deciden crear un negocio. Se evidencia de igual modo que para resolver problemas encuentran el apoyo familiar como la mejor opción y que tienen claro que el entorno es cambiante; por lo tanto, se requiere una mayor disposición al cambio y aceptación del riesgo.

\section{Conclusiones}

El emprendedor se puede definir como un innovador que crea incertidumbre o alguien que aprovecha una oportunidad (Schumpeter, 1934). Diversos estudios se han enfocado en analizar diversos factores que se relacionan con la intención emprendedora. Así, la preferencia que tiene una persona para ser emprendedora y no empleada se ve afectada por diversas variables, como características personales y factores del entorno (Guzmán y Santos, 2001).

La educación es uno de los factores más estudiados en los últimos años, por ser de relevancia para las mujeres al permitirles alcanzar sus metas personales y laborales (Ortiz Casallas, 2017). Es por ello que la formación en las universidades basada en el desarrollo de competencias en emprendimiento contribuye al fortalecimiento e incremento de la intención emprendedora en los estudiantes, especialmente en las mujeres, para promover comportamientos empresariales y superar el techo de cristal que afrontan muchas de ellas (Diaz Bretones y Radrigán, 2018).

El presente trabajo contribuye al estado del arte del emprendimiento femenino, al encontrar diferencias significativas en la relación que existe entre la intención emprendedora y las características personales y del entorno de las estudiantes de las cuatro facultades en una Universidad de Bucaramanga, Colombia. Así, se evidencia que la intención emprendedora de las alumnas adscritas a la Facultad de Ciencias Económicas, Administrativas y Contables aumenta con la disciplina. Esto puede deberse al fuerte deseo de crear un negocio durante o al finalizar su formación profesional y para ello consideran que deben tener una actitud persistente para alcanzar sus metas (Fontaines Ruíz et al., 2015). En relación con las estudiantes de la Facultad de Comunicación Artes y Diseño, la intención emprendedora es mayor en aquellas estudiantes con disposición a asumir riesgos y con experiencia en negocios, debido a su predisposición positiva a la creación de empresas al considerarse capaces de tener éxito en sus iniciativas emprendedoras (Castelao Naval et al., 2015).

En el caso de las universitarias que cursan un programa de la Facultad Ciencias Sociales y Humanas, su intención emprendedora se correlaciona con las redes de apoyo (Universidad y familiares) y la situación actual provocada por la COVID-19, ya que consideran la Universidad como un espacio en el cual pueden adquirir la capacitación idónea en temas de emprendimiento, y sus familiares como el apoyo fundamental para desarrollar sus sueños a pesar del actual entorno económico (Cortez y Da Silva Veiga, 2019; Marulanda Valencia et al., 2014). Para la Facultad de Ingenierías, no fue posible dar a conocer resultados del análisis de correlación entre la variable dependiente e independientes por la multicolinealidad entre estas variables.

Asimismo, los resultados permiten concluir que las estudiantes de las cuatro facultades se consideran capaces de sacar un negocio adelante y para ello cuentan con ciertas habilidades como trabajo en equipo y escucha. A pesar de ello, un poco menos de la mitad manifiestan que no poseen los conocimientos técnicos para elaborar un determinado producto o servicio. Esto puede deberse a que tienen poca experiencia en emprender y falta de acceso a redes de apoyo (socios estratégicos) como escenarios de motivación y conocimiento para el diseño de la propuesta de valor. Igualmente, se concluye que el apoyo familiar es un factor de relevancia para la intención emprendedora de las estudiantes universitarias, y la actual situación económica provocada por la pandemia de la COVID-19 la perciben como una oportunidad para emprender y mejorar su calidad de vida.

Así, teniendo en cuenta los resultados obtenidos de esta investigación, se recomienda a la IES fortalecer la disciplina como la habilidad de plantearse una meta y persistir hasta alcanzarla, y mejorar las competencias orientadas al reconocimiento de las necesidades de un determinado nicho de mercado. Esto, debido a que resultan cruciales para que estas mujeres se empoderen en el ámbito laboral y económico al materializar sus ideas de negocio, con lo que logran el crecimiento esperado y la supervivencia empresarial de sus proyectos a lo largo del tiempo. En este orden de ideas, es importante que la Universidad desarrolle productos de educación no formal para fortalecer esta habilidad y competencia. Igualmente, es necesario crear actividades académicas enfocadas en la innovación empresarial, la adquisición de conocimientos técnicos para elaborar un determinado producto o servicio, el marketing digital, la 
Youseline Garavito-Hernández, Sebastián García-Méndez, Johana Andrea Calderón-Campos

La intención emprendedora y su relación con las características personales y del contexto de las mujeres universitarias

optimización de recursos, la normatividad legal vigente y conocimientos de los diferentes tipos de financiación.

Otros aspectos relevantes que se recomienda a la IES para promover la intención emprendedora y favorecer el desarrollo de un entorno promisorio para la generación de ideas de negocio es el desarrollo de actividades de proyección social como son seminarios, talleres grupales y foros enfocados en fortalecer la interacción entre estudiantes, posibles socios estratégicos y familiares. Estos actores se consideran claves para promover la intención emprendedora en las mujeres universitarias, para que en un futuro cercano hagan realidad sus iniciativas de negocios al romper el techo de cristal que afrontan muchas de ellas. Como futura línea de investigación, se recomienda replicar este estudio en una muestra representativa de varias universidades en Colombia para identificar cuáles otras posibles estrategias se pueden desarrollar en el interior de las universidades, para empoderar a las mujeres en el tema de emprendimiento.

\section{Referencias}

Berdugo Cotera, E. y Gámex Gutiérrez, J. (2015). Mujeres empresarias en Iberoamérica. Casos: España, México y Colombia. Universidad de La Salle.

Borjas de Xena, L. (2012). La educación empresarial en instituciones de educación superior venezolanas. Estudios Gerenciales, 28(125), 51-58. https://doi. org/10.1016/S0123-5923(12)70007-4

Bosma, N., Hill, S., lonescu, A., Kelley, D., Levie, J. y Tarnawa, A. (2020). Global Entrepreneurship Monitor 2019/2020 Global Report. Global Entrepreneurship Research Association. https://www.gemconsortium.org/ report

Bosma,N.yKelley,D.(2019).GlobalEntrepreneurshipMonitor 2018/2019. Global Entrepreneurship Research Association. https://www.gemconsortium.org/ report/gem-2018-2019-global-report

Castelao Naval, M. O., González Pascual, J. L., Jordán Ramos, A. y Ruiz Pomada, A. (2015). Universidad y emprendimiento. Intención emprendedora en estudiantes de universidades privadas madrileñas. Revista de Investigación en Educación, 13(2), 187-205.

Chirinos Araque, Y. del V., Meriño Cordoba, V. H. y Martinez de Meriño, C. Y. (2018). Emprendimiento sostenible: una opción para el crecimiento local. I+D Revista de Investigaciones, 11(1), 116-128. https:// doi.org/10.33304/revinv.v11n1-2018009

Clancy, S. (2007). ¿Por qué no hay más mujeres en la cima de la escala corporativa: debido a estereotipos, a diferencias biológicas o a escogencias personales? Revista Latinoamericana de Administración, 38, 1-8.

Cliff, J. E. (1998). Does One Size Fit All? Exploring the Relationship Between Attitudes Towards Growth, Gender, and Business Size. Journal of Business Venturing, 13(6), 523-542. https://doi.org/10.1016/ S0883-9026(97)00071-2

Cortez, P. A. y Da Silva Veiga, H. M. (2019). Intención emprendedora en la universidad. Ciencias Psicológicas, 13(1), 134-149. https://doi.org/10.22235/ cp.v13i1.1815

Cuervo García, Á., Pérez Díaz, J. M., Ripollés-Melia, M., Sastre Castillo, M. Á., Urbano Pulido, D. y Valdaliso Gago, J. (2005). La empresa y el espíritu emprendedor de los jóvenes. Ministerio de Educación, Cultura y Deporte, y Secretaría General Técnica.

Departamento Administrativo Nacional de Estadísticas DANE. (2018). Comunicado de prensa. Informes especiales de mercado laboral trimestre móvil mayojulio 2018. https://bit.ly/2JC6y4A

Diaz Bretones, F. y Radrigán, M. (2018). Actitudes hacia el emprendimiento: El caso de estudiantes universitarios chilenos y españoles. CIRIEC-España, Revista de Economía Pública, Social y Cooperativa, 94, 11-30. https://doi.org/10.7203/CIRIEC-E.94.12668

Eagly, A. H. y Wood, W. (1991). Explaining Sex Differences in Social Behavior: A Meta-Analytic Perspective. Personality and Social Psychology Bulletin, 17(3), 306315. https://doi.org/10.1177/0146167291173011

Fontaines Ruíz, T., Palomo de Rivero, M. y Velásquez, M. V. (2015). Resiliencia como componente de la actitud emprendedora de los jóvenes universitarios. Revista Educación y Desarrollo, 9(1), 160-183.

Forero-Bernal, L. A. y Durán-Duarte, L. K. (2019). Aportes a la construcción del estado de arte del emprendimiento femenino en Colombia. Revista Escuela de Administración de Negocios, 86, 77-92. https://doi.org/10.21158/01208160.n86.2019.2291

Galindo, M. S. y Herrera, S. L. (2017). La categoría de género en la investigación y producción de conocimiento en enfermería en Iberoamérica: Aportes para el debate. La Ventana. Revista de Estudios de Género, 5(46), 177-201. https://doi.org/10.32870/ Iv.v5i46.6041

Garavito-Hernández, Y., Córdoba-Campos, J. A. y ArdilaSequeda, R. (2020). Iniciativa emprendedora del recurso humano inactivo en el Establecimiento Penitenciario de Mediana Seguridad y Carcelario de Bucaramanga. I+D Revista de Investigaciones, 
Youseline Garavito-Hernández, Sebastián García-Méndez, Johana Andrea Calderón-Campos La intención emprendedora y su relación con las características personales y del contexto de las mujeres universitarias

15(1), 57-66. https://doi.org/10.33304/revinv.v15n12020006

García-Cabrera, A. M., Déniz-Déniz, M. de la C. y Cuéllar-Molina, D. (2015). Inteligencia emocional y emprendimiento: posibles líneas de trabajo. Cuadernos de Administración, 28(51), 65-101. https:// doi.org/10.11144/Javeriana.cao28-51.ieep

García, J. C., Villaseca, D. y González, S. (2018). Emprendimiento femenino y financiación social: Un estudio comparado. REVESCO. Revista de Estudios Cooperativos, 132, 97-121. https://doi.org/10.5209/ reve.65974

George Reyes, C. E. y Trujillo Liñan, L. (2018). Aplicación del método delphi modificado para la validación de un cuestionario de incorporación de las TIC en la práctica docente. Revista Iberoamericana de Evaluación Educativa, 11(1), 113-135. https://doi. org/10.15366/riee2018.11.1.007

Gómez Mujica, A. y Acosta Rodríguez, H. (2003). Acerca del trabajo en grupos o equipos. ACIMED, 11(6).

Gómez Núñez, L., López Gómez, S., Hernández Vargas, N., Galvis Maldonado, M., Parra Bernal, L., Matiz Bulla, F., Varela Villegas, R., Moreno Barragán, J., Pereira Laverde, F., Arias, A., García Cediel, G. y Martínez Carazo, P. (2017). GEM Colombia. Estudio de la actividad empresarial 2017. https://gemcolombia. org/

Guzmán, J. y Santos, F. (2001). The Booster Function and the Entrepreneurial Quality: An Application to the Province of Seville. Entrepreneurship \& Regional Development, 13(3), 211-228. https://doi. org/10.1080/08985620110035651

Henry, C., Foss, L. y Ahl, H. (2016). Gender and Entrepreneurship Research: A Review of Methodological Approaches. International Small Business Journal, 34(3), 217-241. https://doi. org/10.1177/0266242614549779

Hernandez Sampieri, R., Fernandez Collado, C. y Baptista Lucio, P. (2006). Metodología de la investigación (1 ${ }^{\text {a }}$ ed.). McGraw-Hill/Interamericana de Editores.

Kepler, E. y Shane, S. (2007). Are Male and Female Entrepreneurs Really That Different? Small Business Research Summary. http://www.pw.seipa.edu. $\mathrm{pl} / \mathrm{s} / \mathrm{p} / \mathrm{artykuly/90/909/Female \quad Entrepreneurs}$ Different Shane 2007.pdf

Kurniawan, M. E. H., Yudoko, G., Basri, M. H. y Umbara, A. N. (2019). Do Entrepreneurship Students Have an Intention to Become an Entrepreneur? Journal of Entrepreneurship Education, 22(2), 1-14.
Laukkanen, M. (2000). Exploring Alternative Approaches in High-Level Entrepreneurship Education: Creating Micromechanisms for Endogenous Regional Growth. Entrepreneurship \& Regional Development, 12(1), 25-47. https://doi. org/10.1080/089856200283072

Marlow, S. y Patton, D. (2005). All Credit to Men? Entrepreneurship, Finance, and Gender. Entrepreneurship Theory and Practice, 29(6), 717-735. https://doi.org/10.1111/j.1540-6520.2005.00105.x

Martínez, C. y Rubio Bañón, A. (2020). Emprendimiento en épocas de crisis: Un análisis exploratorio de los efectos de la COVID-19. Small Business International Review, 4(2), 53-66. https://doi.org/10.26784/sbir. v4i2.279

Marulanda Valencia, F. Á., Montoya Restrepo, I. A. y Vélez Restrepo, J. M. (2014). Aportes teóricos y empíricos al estudio del emprendedor. Cuadernos de Administración, 30(51), 89-99.

Mun, E. y Jung, J. (2018). Policy Generosity, Employer Heterogeneity, and Women's Employment Opportunities: The Welfare State Paradox Reexamined. American Sociological Review, 83(3), 508-535. https://doi.org/10.1177/0003122418772857

Naldi, L., Baù, M., Ahl, H. y Markowska, M. (2019). Gender (In)Equality Within the Household and Business Start-Up Among Mothers. Small Business Economics, 1-16. https://doi.org/10.1007/s11187-019-00275-1

OECD. (2017). Education at a Glance 2017: OECD Indicators. OECD Publishing. https://doi.org/10.1787/eag-2017en

Ortiz Casallas, E. M. (2017). Discursos y género, en relatos de hombres y mujeres. Agora U.S.B., 17(1), 191. https://doi.org/10.21500/16578031.2818

Ovalles-Toledo, L., Moreno, Z., Olivares, M.y Silva, H. (2018). Habilidades y capacidades del emprendimiento: un estudio bibliométrico. Revista Venezolana de Gerencia, 23(81), 217-234.

Pabón, R. (2011). Estudio de actitudes emprendedoras con profesionales que crearon empresa. Revista Escuela de Administración de Negocios, 71, 70-83.

Pimienta, M. L. (2008). Comunicación organizacional: Los stakeholders legitiman a la organización. Chasqui: Revista Latinoamericana de Comunicación, 103, 8083.

Pinillos Costa, M. J., Reyes Recio, L. E. y Soriano Pinar, I. (2007). Actividad emprendedora en países de alto desarrollo. XXI Congreso Anual AEDEM, Universidad 
Youseline Garavito-Hernández, Sebastián García-Méndez, Johana Andrea Calderón-Campos

La intención emprendedora y su relación con las características personales y del contexto de las mujeres universitarias

Rey Juan Carlos, 1-15. https://dialnet.unirioja.es/ servlet/libro?codigo $=275423$

Robb, A. M. (2002). Entrepreneurial Performance by Women and minorities: The Case of New Firms. Journal of Developmental Entrepreneurship, 7(4), 383-397.

Roy-García, I., Rivas-Ruiz, R., Pérez-Rodríguez, M. y Palacios-Cruz, L. (2019). Correlación: No toda correlación implica causalidad. Revista Alergia México, 66(3), 354-360. https://doi.org/10.29262/ ram.v66i3.651

Rueda, I., Sánchez, L., Herrero, Á., Blanco, B. y FernándezLaviada, A. (2013). ¿Existen niveles adecuados de formación y financiación que incentiven la intención emprendedora? Revista FIR, FAEDPYME International Review, 2(3), 28-38.

Sabarwal, S. y Terrell, K. (2008). Does Gender Matter For Firm Performance? Evidence From Eastern Europe And Central Asia (Policy Research Working Papers). The World Bank. https://doi.org/10.1596/18139450-4705

Sánchez García, J. C., Ward, A., Hernández, B. y Florez, J. L. (2017). Educación emprendedora: Estado del arte. Propósitos y Representaciones, 5(2), 401-473. https:// doi.org/10.20511/pyr2017.v5n2.190

Santiago Martínez, V. y Márquez Rodríguez, P. (2018). Perfil de los empresarios colombianos: Estudio de la Tubería Empresarial 2006-2011, a partir de los datos del Global Entrepreneurship Monitor (GEM). En R. Varela Villegas y J. A. Moreno Barragán (eds.), Cultura empresarial en América Latina (pp. 43-62). Centro de Desarrollo del Espíritu Empresarial (CDEE) y Editorial Universidad Icesi.

Sastre, R. F. (2013). La Motivación emprendedora y los Factores que contribuyen con el éxito del emprendimiento. Ciencias Administrativas, 1, 1-10.

Schumpeter, J. A. (1934). The Theory of Economic Development. Harvard University Press.

Thébaud, S. (2010). Institutions, Cultural Beliefs and the Maintenance of Gender Inequality in Entrepreneurship Across Industrialized Nations. In SSRN Electronic Journal. https://doi.org/10.2139/ ssrn.1664554

Tinkler, J. E., Whittington, K., Ku, M. C. y Davies, A. R. (2015). Gender and Venture Capital DecisionMaking: The Effects of Technical Background and Social Capital on Entrepreneurial Evaluations. Social Science Research, 51, 1-16. https://doi.org/10.1016/j. ssresearch.2014.12.008
Valencia Arias, A., Montoya Restrepo, I. y Montoya Restrepo, A. (2016). Intención emprendedora en estudiantes universitarios: Un estudio bibliométrico. Intangible Capital, 12(4), 881-922. https://doi.org/10.3926/ic.730

Van Gelderen, M., Thurik, R. y Bosma, N. (2006). Success and Risk Factors in the Pre-Startup Phase. Small Business Economics, 26(4), 319-335. https://doi. org/10.1007/s11187-004-6837-5

Zambrano-Vargas, S. M. y Vázquez-García, A. W. (2019). Algunas perspectivas teóricas para el estudio del emprendimiento y el género. Saber, Ciencia y Libertad, 14(1), 159-170. https://doi. org/10.18041/2382-3240/saber.2019v14n1.5216. 
Youseline Garavito-Hernández, Sebastián García-Méndez, Johana Andrea Calderón-Campos La intención emprendedora y su relación con las características personales y del contexto de las mujeres universitarias

\section{Anexo 1. Dimensiones Variables dependientes e independientes}

\begin{tabular}{|c|c|c|c|}
\hline Variable & Dimensión & Preguntas & Variable por pregunta \\
\hline \multirow{4}{*}{ Variable dependiente } & \multirow{4}{*}{ Intención emprendedora } & $\begin{array}{l}\text { Soy una persona que prefiere crear un negocio } \\
\text { que buscar un empleo }\end{array}$ & Intención emprendedora \\
\hline & & Me surgen ideas para emprender un negocio & Intención emprendedora \\
\hline & & $\begin{array}{l}\text { Al terminar la condena tengo planeado } \\
\text { emprender una idea de negocio }\end{array}$ & Intención emprendedora \\
\hline & & Quiero emprender un negocio desde la cárcel & Intención emprendedora \\
\hline \multirow{29}{*}{ Variables independientes } & \multirow{24}{*}{ Características personales } & Edad & Demográfica \\
\hline & & Estado civil & Demográfica \\
\hline & & Estrato social & Demográfica \\
\hline & & Hijos & Demográfica \\
\hline & & Semestre & Semestre \\
\hline & & Programa de pregrado & Programa académico \\
\hline & & Me gustaría asumir el riesgo de crear un negocio & Asumir riesgos \\
\hline & & Tengo experiencia en emprender un negocio & Experiencia \\
\hline & & Soy una persona que le gusta trabajar en equipo & Trabajo en equipo \\
\hline & & $\begin{array}{l}\text { Me gusta que otras personas me den su opinión } \\
\text { a mis ideas. }\end{array}$ & Capacidad de escucha \\
\hline & & $\begin{array}{l}\text { ¿Usted ha recibido capacitación en temas de } \\
\text { emprendimiento? }\end{array}$ & Formación \\
\hline & & $\begin{array}{l}\text { Tengo conocimiento para elaborar un producto } \\
\text { con el que podría montar un negocio }\end{array}$ & Formación \\
\hline & & $\begin{array}{l}\text { Me considero una persona capaz de sacar un } \\
\text { negocio adelante. }\end{array}$ & Locus de control interno \\
\hline & & $\begin{array}{l}\text { Cuando tengo un problema, siempre busco otras } \\
\text { alternativas de solución }\end{array}$ & Resolución de problemas \\
\hline & & $\begin{array}{l}\text { Creo que montar un negocio es un proyecto para } \\
\text { toda la vida }\end{array}$ & Pensamiento a largo plazo \\
\hline & & $\begin{array}{l}\text { Emprender un negocio es para obtener } \\
\text { beneficios económicos }\end{array}$ & Coste de oportunidad \\
\hline & & $\begin{array}{l}\text { Definitivamente, ser emprendedor mejoraría mi } \\
\text { calidad de vida }\end{array}$ & Necesidad de logro \\
\hline & & $\begin{array}{l}\text { Soy una persona capaz de controlar mis } \\
\text { emociones }\end{array}$ & $\begin{array}{c}\text { Habilidades del ser - inteligencia } \\
\text { emocional }\end{array}$ \\
\hline & & $\begin{array}{l}\text { Me considero una persona ordenada cuando } \\
\text { realizo una actividad }\end{array}$ & Habilidades del ser - Disciplina \\
\hline & & $\begin{array}{l}\text { Soy una persona que no se rinde ante las } \\
\text { dificultades y lo intento de nuevo }\end{array}$ & Habilidades del ser - resiliencia \\
\hline & & $\begin{array}{l}\text { No tengo problema en obedecer las órdenes de } \\
\text { mis superiores }\end{array}$ & $\begin{array}{l}\text { Habilidades interpersonales - } \\
\text { Respeto }\end{array}$ \\
\hline & & $\begin{array}{l}\text { Me considero una persona que transmite con } \\
\text { claridad sus ideas a los demás }\end{array}$ & $\begin{array}{l}\text { Habilidades interpersonales - } \\
\text { Comunicación }\end{array}$ \\
\hline & & $\begin{array}{l}\text { Me considero una persona que sabe delegar el } \\
\text { trabajo a quien corresponda }\end{array}$ & $\begin{array}{l}\text { Habilidades interpersonales - } \\
\text { Delegación }\end{array}$ \\
\hline & & $\begin{array}{l}\text { Soy una persona muy entusiasta cuando debo } \\
\text { realizar mis labores }\end{array}$ & $\begin{array}{l}\text { Habilidades interpersonales - } \\
\text { Pasión }\end{array}$ \\
\hline & \multirow{3}{*}{$\begin{array}{l}\text { Factores del entorno } \\
\text { personal del emprendedor }\end{array}$} & $\begin{array}{l}\text { Tengo familiares que han sido emprendedores o } \\
\text { poseen un negocio }\end{array}$ & Familia \\
\hline & & $\begin{array}{l}\text { Mi familia me apoyaría si quisiera iniciar un } \\
\text { negocio }\end{array}$ & Familia \\
\hline & & $\begin{array}{l}\text { Tengo amigos y contactos que serían socios si } \\
\text { decido crear un negocio }\end{array}$ & Redes de apoyo \\
\hline & \multirow{2}{*}{$\begin{array}{l}\text { Factores del entorno } \\
\text { global del emprendedor }\end{array}$} & $\begin{array}{l}\text { La actual crisis provocada por la COVID-19 } \\
\text { representa una oportunidad para emprender }\end{array}$ & Programas gubernamentales \\
\hline & & $\begin{array}{l}\text { ¿Qué tipo de capacitación le gustaría recibir en la } \\
\text { universidad para poder crear un negocio? }\end{array}$ & Universidad \\
\hline
\end{tabular}

